\title{
ResearchOnline@JCU
}

This is the author-created version of the following work:

Jillings, E.K.P, Squires, R.A., Azarpeykan, S., and Lopez-Villalobos, N. (2019)

Does blood contamination of urine compromise interpretation of the urine protein to creatinine ratio in dogs?. New Zealand Veterinary Journal, 67 (2) pp. 74-78.

Access to this file is available from:

https://researchonline.jcu.edu.au/56540/

(C) 2019 New Zealand Veterinary Association. In accordance with the publisher's policies, the Accepted Manuscript version of this paper is available Open Access from ResearchOnline@JCU following the expiry of the embargo period on 16 January 2020.

Please refer to the original source for the final version of this work: 


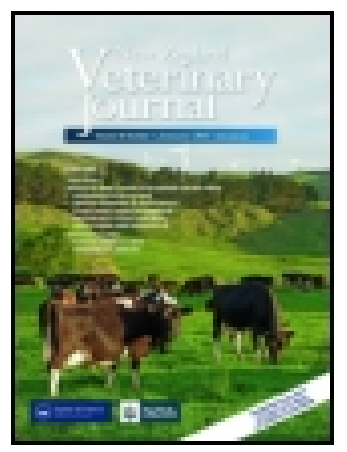

New Zealand Veterinary Journal

\section{Does blood contamination of urine compromise interpretation of the urine protein to creatinine ratio in dogs?}

\section{EKP Jillings, RA Squires, S Azarpeykan \& N Lopez-Villalobos}

To cite this article: EKP Jillings, RA Squires, S Azarpeykan \& N Lopez-Villalobos (2018): Does blood contamination of urine compromise interpretation of the urine protein to creatinine ratio in dogs?, New Zealand Veterinary Journal, DOI: 10.1080/00480169.2018.1556129

To link to this article: https://doi.org/10.1080/00480169.2018.1556129

Accepted author version posted online: 05

Dec 2018.

Submit your article to this journal $\sqsubset$

View Crossmark data ¿ 
Publisher: Taylor \& Francis \& New Zealand Veterinary Association

Journal: New Zealand Veterinary Journal

DOI: $10.1080 / 00480169.2018 .1556129$

Scientific Article

\title{
Does blood contamination of urine compromise interpretation of the urine protein to creatinine ratio in dogs?
}

\section{EKP Jillings ${ }^{*}$, RA Squires ${ }^{\dagger}$, S Azarpeykan*, N Lopez-Villalobos}

* School of Veterinary Science, Massey University, Private Bag 11-222, Palmerston North, 4442, New Zealand

${ }^{\dagger}$ College of Public Health, Medical and Veterinary Sciences, James Cook University, 1 Solander Drive, Townsville, Queensland 4811, Australia.

§ Author for correspondence. Email: E.Jillings@massey.ac.nz

\begin{abstract}
AIMS: To determine the effect of contamination of urine with $0-5 \%$ blood, varying in haematocrit and protein concentrations, on the urine protein to creatinine ratio (UPC) in dogs, and to determine whether the colour of urine can be used to aid interpretation of UPC results.
\end{abstract}

METHODS: Urine samples were collected by free catch from 18 dogs, all of which had UPC $<0.2$. Venous blood samples were also collected from each dog, and the blood from each dog was added to its own urine to produce serial concentrations of $0.125-5 \%$ blood. The colour of each urine sample was recorded by two observers scoring them as either yellow, peach, orange, orange/red or red. Protein and creatinine concentrations were determined, and dipstick analysis and sediment examination was carried out on each sample. Based on colour and dipstick analysis, samples were categorised as either having microscopic, macroscopic or gross haematuria. A linear mixed model was used to examine the effect of blood contamination on UPC.

RESULTS: The uncontaminated urine of all 18 dogs had a UPC $<0.2$. Adding blood to the urine samples resulted in an increase in UPC at all contamination concentrations compared to the noncontaminated urine $(\mathrm{p}<0.001)$. None of the 54 samples with microscopic haematuria had UPC $>0.5$. For 108 samples with macroscopic haematuria the UPC was $>0.5$ in 21 samples $(19.4$ (95\% $\mathrm{CI}=13.1-27.9) \%)$, and for 54 samples with gross haematuria $39(72(\mathrm{CI}=59.1-82.4) \%)$ had a UPC $>0.5$. No samples had a UPC $>2.0$ unless the blood contamination was $5 \%$ and only $3 / 18(17 \%)$ samples at this blood contamination concentration had a UPC $>2.0$. 
CONCLUSIONS AND CLINICAL RELEVANCE: This study showed that while blood contamination of $\geq 0.125 \%$ does increase the UPC, if the urine remains yellow (microscopic haematuria), then there is negligible chance that a UPC $>0.5$ will be solely due to the added blood. In that scenario, attributing the proteinuria present to the haematuria in the sample would be inappropriate. However blood contamination that results in discolouration of the urine sample from yellow to red (indicating macroscopic or gross haematuria) could increase the UPC above the abnormal range and would need to be considered as a differential for the proteinuria. Thus knowledge of urine colour, even if limited to simple colour scores (yellow, discoloured, red) could be utilised to aid interpretation of the UPC in samples with haematuria.

KEY WORDS: Proteinuria, urine protein to creatinine ratio, haematuria, blood contamination, urine colour

$\begin{array}{ll}\text { NZVP } & \text { New Zealand Veterinary Pathology } \\ \text { UPC } & \text { Urine protein to creatinine ratio } \\ \text { USG } & \text { Urine specific gravity }\end{array}$

\section{Introduction}

Proteinuria, an increase in the concentration of protein in the urine, is seen commonly in dogs for both pathological and physiological reasons, which are often categorised as pre-renal, renal, and post-renal (Lees et al. 2005). Pre-renal proteinuria may also be referred to as overflow proteinuria, and reflects a transient increase in the blood concentration and subsequent renal filtration of small protein molecules such as haemoglobin, myoglobin and light chain immunoglobulin (i.e. BenceJones proteins). Renal proteinuria has two subtypes: functional and pathological proteinuria. Functional proteinuria is not associated with renal pathology but rather reflects altered renal physiology in transient situations including, but not limited to, fever, and strenuous exercise (Lees et al. 2005). Pathological renal proteinuria can be further subtyped into tubular, interstitial and glomerular proteinuria, due to impaired tubular protein resorption, inflammation of the interstitium, and altered glomerular filtration, respectively. Glomerular proteinuria is the most clinically important category and needs to be distinguished from commonly-encountered post renal proteinuria, which is a result of protein (e.g. haemorrhage or inflammatory exudate) being deposited in the urine from any part of the urinary tract distal to the renal pelvis (Lees et al. 2005). 
Proteinuria is often first detected as an increased protein reading on a urine dipstick, which is usually then confirmed and quantified by use of the urine protein to creatinine ratio (UPC) (Lees et al. 2005; Harley and Langston 2012; Vaden and Elliott 2016). The UPC measured in a single canine urine sample has been shown to be a reliable method to assess total protein measured in urine over a 24 hour period (White et al. 1984; Jergens et al. 1987). Along with its utility for determination of proteinuria, the UPC has also become an important prognostic tool in chronic kidney disease (Jacob et al. 2005; Vilhena et al. 2015; Jepson 2016).

Proteinuria is confirmed in dogs with UPC persistently $>0.5$ (Lees et al. 2005; Vaden and Elliott 2016). Dogs with UPC $<0.2$ are considered non-proteinuric, and with UPC of $0.2-0.5$ are considered borderline (Vaden and Elliott 2016). In the absence of evidence of pre-renal or postrenal proteinuria, a UPC $>2.0$ is suggestive of glomerular disease (Lees et al. 2005; Cianciolo et al. 2016; Vaden and Elliott 2016).

Interpretation of the UPC should always be completed in conjunction with a review of concurrent urinalysis results, including sediment examination, as it has been reported that proteinuria can be detected in urine samples that show evidence of inflammation or haemorrhage (Grauer et al. 1985; Bagley et al. 1991). In such cases, it is unclear whether the protein present is due entirely to the haemorrhage or inflammation itself, as the UPC cannot be used to distinguish between causes of proteinuria (Jansen and Lumsden 1985; Bagley et al. 1991). On the basis of these findings, the practice of attributing proteinuria to haematuria in a sample, or declining to assess UPC if microscopic haemorrhage was present, became commonplace. It was considered that samples with evidence of haematuria and or inflammation could not be reliably assessed for proteinuria, but that in the absence of inflammation or haematuria, persistent proteinuria indicates renal disease, often glomerular (Center et al. 1985; Brunker 2005; Grauer 2005).

Two studies have investigated the effect of blood contamination on the UPC in a single urine sample with somewhat differing results. In the first, a single canine urine sample was contaminated with large amounts of blood to give final blood contamination concentrations of 10, 20, 50 and 75\% (1:10 to $3: 4)$ (Bagley et al. 1991). These contamination concentrations all resulted in gross blood contamination and substantially increased UPC. The authors concluded that gross blood contamination of a urine sample increases the UPC, and suggested that it remained to be demonstrated whether low levels of contamination with blood would substantially affect the diagnostic usefulness of the UPC (Bagley et al. 1991).

The second report was also of blood contamination of a single canine urine sample, however with very low contamination ranging from $0.0039-0.25 \%$ (1:25600 to 1:400) (Vaden et al. 2004). In this 
study the UPC did not exceed 0.3 at any blood contamination concentration, even when the urine was described to be discoloured pink to red. The authors concluded that macroscopic haematuria may not increase the UPC ratio (Vaden et al. 2004). These two studies, while similar in design, varied markedly in the concentration of blood contamination, with neither including concentrations of $0.25-10 \%$, which may coincide with levels of haematuria commonly seen in companion animal practice. These results identify the need for a study which assesses blood contamination of urine at concentrations bridging those of the previous two studies, in multiple dogs.

The objectives in this study were to determine the effect of contamination of urine with $0-5 \%$ blood, varying in haematocrit and protein concentrations, on the UPC in dogs, and to determine whether the colour of urine can be used to aid interpretation of UPC results.

\section{Materials and methods}

Urine samples were collected by free catch from dogs owned bystaff of the Massey University Veterinary Teaching Hospital (Palmerston North, NZ). Only dogs that had a UPC $<0.2(n=18)$ were included in the study.

Immediately after urine collection, a blood sample from each dog was collected and transferred into two blood collection tubes, one containing no additive and one containing EDTA (BD Vacutainer, BD, Franklin Lakes, NJ, USA). A complete blood count (Advia 120, Bayer Healthcare, Leverkusen, Germany) and serum biochemistry profile (P800 Chemistry Module of Hitachi Serum Work Area, Hitachi, Tokyo, Japan) were carried out for each dog by New Zealand Veterinary Pathology (NZVP, Palmerston North, NZ) to rule out underlying disease.

To simulate the effect of naturally occurring haematuria, the urine sample from each dog was serially contaminated with the anticoagulated blood from the same dog to prepare 12 standardised samples with blood contamination concentrations measured on a volume/volume basis ranging from $0-5 \%$ of the total urine volume. As the volume of urine collected from each dog differed, in order to maximise the sample volumes for analysis, variable volumes of urine and blood were mixed to achieve the final standard contamination concentrations for each dog. The urine and blood were mixed in $5 \mathrm{~mL}$ polystyrene culture tubes (Interlab, Wellington, NZ).

The colour of each urine sample was recorded by two observers who were unaware of the blood contamination concentration of each urine sample. These observers assigned each sample a score from 1 to 5 where yellow $=1$, peach $=2$ orange $=3$, orange $/$ red $=4$ ) and red $=5$. To indicate the overall urine colour for each urine contamination concentration, the median colour score for each urine contamination concentration was calculated from the colour observations of all $18 \mathrm{dogs}$. 
Microscopic sediment examination (Stockham and Scott 2008) was performed and urine specific gravity (USG) was assessed on each sample. Dipstick biochemistry read on an automated urine dipstick analyser (Combur 10 Test UX, Roche Diagnostics, Penzberg, Germany) was performed on each non-centrifuged urine sample.

Within 30 minutes of adding blood to the urine samples, the urine protein and creatinine concentrations were assessed using a benzethonium chloride assay (Urine/CSF protein method, Roche Diagnostics) (Iwata and Nishikaze 1979; Luxton et al. 1989), and the Jaffe method (Creatinine Method, Roche Diagnostics) (Bartels et al. 1972), respectively.

For the purposes of this study, the term microscopic haematuria was used to denote yellow urine samples which were positive on the urine dipstick for blood, and contained greater than normal numbers of erythrocytes, i.e. $>5$ per $400 x$ high power field (Vaden et al. 2004), in the sediment. Macroscopic haematuria was defined as urine samples which were visibly discoloured from yellow, but did not appear red and were positive on the urine dipstick for blood, and contained greater than normal numbers of erythrocytes in the sediment. Gross haematuria was used to describe visibly red urine samples that were positive on the urine dipstick for blood and contained greater than normal numbers of erythrocytes in the sediment.

This study was approved by the Massey University Animal Ethics Committee (Palmerston North, NZ).

\section{Statistical analyses}

A linear mixed model was used to examine the effect of blood contamination on UPC, including the fixed effects of percentage blood contamination and sex of dog, the interaction between percentage contamination and sex, and the random effect of dog to account for repeated measures in the same dog. A heterogeneous yariance was accounted for across contamination concentrations. The LSM of the UPC for each of the contamination percentages were compared to the LSM of the uncontaminated urine using the Bonferroni adjustment to correct for multiple comparisons.

To determine whether urine colour can be used to aid interpretation of UPC results, each urine sample was categorised using the three categories of none or microscopic haematuria, macroscopic haematuria, and gross haematuria, described above. The percentage of urine samples within each category with UPC $>0.5$ (indicating proteinuria) was then calculated, and 95\% CI for the percentages were calculated using the Wilson interval method (Brown et al. 2001).

To determine whether the varying haematocrit and concentrations of protein in blood used to contaminate the urine affected the difference in UPC between samples containing 0 and $5 \%$ blood 
contamination (UPC difference), regression equations were used to estimate the regression coefficient for haematocrit and concentrations of protein in blood on the UPC difference for each dog. Analyses were carried out using SAS version 9.4 (Statistical Analysis System; SAS Institute Inc., Cary, NC, USA).

\section{Results}

Eighteen dogs of varying breeds, sex and ages with a UPC $<0.2$ were included in the study. There were 10 female dogs and eight male dogs. Seven of the dogs were aged 1-4 years, six of the dogs were aged 5-9 years while the remaining five dogs were aged 10-13 years. While 15 of the dogs were apparently well, one dog had diabetes mellitus, and two dogs had pruritic dermatitis.

Baseline chemistry and haematology was assessed on all dogs. The total protein concentrations in plasma for all dogs were within the reference interval of 52-75 g/L. The haematocrit of 16 dogs was within the reference interval of $0.37-0.55 \mathrm{~L} / \mathrm{L}$ (all reference intervals provided by NZVP), and the haematocrit of the remaining two dogs was $0.34 \mathrm{~L} / \mathrm{L}$ and $0.58 \mathrm{~L} / \mathrm{L}$.

A complete urinalysis was conducted for all dogs. The USG ranged from 1.018-1.052, it was 1.018 in one dog at, between 1.020-1.029 in six dogs and $\geq 1.030$ in the remaining 11 dogs. No evidence of inflammation or other abnormalities was observed in the sediment of the uncontaminated urine of any dog. Urinary dipstick analysis was negative for blood and protein for 16 dogs. The urine of one dog had negative blood and 1+ protein on urine dipstick analysis, with a USG of 1.045, and for another dog had 1+ blood and negative protein by dipstick analysis, with a USG of 1.035 . For all urine samples with a blood contamination concentration $\geq 0.125 \%$ the dipstick blood result was the maximum value $(3+)$, and for samples with $\geq 0.5 \%$ blood contamination the urine dipstick protein was $\geq 1+$.

The uncontaminated urine of all 18 dogs had a UPC $<0.2$, with a mean UPC of $0.064(95 \%$ $\mathrm{CI}=0.054-0.074)$. The UPC was assessed in a total of 216 samples; the 12 blood contamination concentrations for all $18 \mathrm{dogs}$. As the blood contamination concentration of the samples increased, the UPC also increased (Figure 1). The LSM UPC of all the contamination concentrations differed from the LSM UPC of the non-contaminated urine $(p<0.001)$. There mean UPC of male and female dogs was similar $(\mathrm{p}=0.649)$, and there was no interaction between sex and percentage contamination $(\mathrm{p}=0.998)$.

As the blood contamination concentration of the samples increased, the sample colour changed from yellow to peach, orange and finally red (Table 1). For all 54 yellow samples, which had either no haematuria or microscopic haematuria, none $(0(95 \% \mathrm{CI}=0-6.6) \%)$ had a UPC $>0.5$ (Table 1$)$. In 
108 samples with macroscopic haematuria (peach to orange-red discolouration) the UPC was $>0.5$ in 21 samples $(19.4(95 \% \mathrm{CI}=13.1-27.9) \%)$. There were 54 samples which were categorised as grossly haematuric, of which $39(72(95 \% \mathrm{CI}=59.1-82.4) \%)$ had a UPC $>0.5$.

No samples had a UPC $>2.0$ unless the blood contamination percentage was 5\% (visibly red) and only $3 / 18(17 \%)$ samples at this blood contamination concentration had a UPC >2.0 (Table 1).

The regression coefficient for the effect of haematocrit on the UPC difference was $3.62\left(\mathrm{R}^{2}=0.14\right.$; $\mathrm{p}=0.132 ;)$ and for concentration of total protein on the UPC difference was $0.02\left(\mathrm{R}^{2}=0.015\right.$; $\mathrm{p}=0.628$ ).

\section{Discussion}

The results of this study showed that adding blood to canine urine samples to obtain final contamination concentrations of $0.125-5 \%$ increased the UPC of the contaminated sample. However, in order to elevate the UPC into the abnormal range $(>0.5)$, in urine samples with previously normal UPC $(<0.2)$, the amount of blood added would need to cause the contaminated sample to appear at least orange in colour (Table 1).

In this study the urine samples were collected by free catch, which has been shown to yield results highly correlated to the UPC results of urine collected by cystocentesis (Beatrice et al. 2010; Marynissen et al. 2017). The decision was made to contaminate the urine sample of each dog with its own blood to more accurately reflect the situation in vivo. Whilst it would have removed a variable in this study to have contaminated all the urine samples with a single, standard blood sample, this would not have reflected the reality that each dog has its own individual blood protein concentration and haematocrit. From the analysis of regression of total protein or haematocrit on the difference in UPC between 0 and 5\% blood contamination, there was no evidence of the UPC difference being affected by the varying haematocrit and total protein concentrations, as shown by the very low $\mathrm{R}^{2}$ value of the regression lines. Adding each dogs own blood to its urine sample did not appear to have a biologically relevant affect on the changes in UPC values.

Adding blood to the urine samples in this study increased the UPC at all contamination concentrations as compared to the non-contaminated samples. Practically however in any urine sample with only microscopic haematuria (yellow urine) due to the blood contamination, no samples had UPC $>0.5$, and the UPC rarely exceeded the normal value of 0.2 (Table 1 ). These findings are supported by that of a previous study in which a single canine urine sample was contaminated with its own blood (55\% packed cell volume ) at a ratio of 1:25,600 (0.0039\%) to 1:400 (0.25\%) (Vaden et al. 2004). Another study reported no significant difference in protein 
concentration between paired urine samples of 14 dogs pre and post catheterisation, despite increased numbers of erythrocytes in the post-catheterisation samples (McCaw et al. 1985). The results of these studies support the findings of the current study which suggest that providing the urine sample is yellow, although there may be microscopic haematuria, the proteinuria as measured by the UPC will not be elevated into the proteinuric range due to the blood present. This suggests that the presence of microscopic haematuria in yellow urine, without concurrent evidence of urinary tract inflammation, will not interfere with the interpretation of UPC results. Therefore the practice of attributing proteinuria to samples, if there is blood present, should be discouraged.

In the present study, when the contaminated urine samples had macroscopic or gross haematuria, the percentage of samples with UPC $>0.5$ ranged from $19-72 \%$. This differs from the findings of Vaden et al. (2004), in which samples that were observed as being red did have higher UPC than the non-contaminated sample, however the UPC never exceeded 0.5. The magnitude of blood contamination required to achieve colour changes in that previous study contrasted markedly with those in our present study. In that previous study, the urine sample with a blood contamination concentration of $0.03 \%$ was observed to be pink (i.e. macroscopic haematuria) while the highest contamination concentration $(0.25 \%)$ was noted as red. In our present study, samples with blood contamination up to and including $0.25 \%$ were assessed as yellow, and were not noted as red until there was $2 \%$ blood contamination or greater. The reason for this discrepancy is not clear.

The assessment of colour is subjective and differences in colour interpretation may exist. In the current study two observers were used, who each completed 216 colour score observations. Their scores were identical for 184 observations, and differed for 32 observations, but in all 32 instances the colour score only differed by one (e.g. 2 vs. 3 or 4 vs. 5). However, when considering the three categories of none/microscopic, macroscopic or gross haematuria there were only seven individual instances out of 216 in which the observers differed in their score.

In the current study, $72 \%$ of grossly haematuric samples (2-5\% blood contamination), had a UPC $>0.5$. Three of the 18 samples with $5 \%$ blood contamination had UPC $>2.0$, which could have been mistakenly classified as severely proteinuric if the urine sample was used to measure UPC. These findings are supported by results of an earlier study in which a single canine urine sample was contaminated with blood ( $42 \%$ packed cell volume) to make up $10-75 \%$ of the total sample volume (Bagley et al. 1991). In that study, all samples appeared red and on microscopic examination the number of erythrocytes per high power field was too numerous to count. The UPC of the samples ranged from 1.5 in the $10 \%$ blood contaminated sample to 30.6 in the $75 \%$ contaminated sample, leading the authors to conclude that gross blood contamination of a urine sample increases the UPC 
(Bagley et al. 1991). However, contrasting results were obtained from the study of Vaden et al. (2004) in which the two urine samples with the highest blood contamination concentration $(0.125$ and $0.250 \%$ ) were reported as visibly red while still having UPC $\leq 0.3$. As previously noted, the colour observations for similar percentages of blood contamination differed markedly between the previous study by Vaden et al. (2004) and our present study. The authors of that study noted that macroscopic haematuria may not elevate the UPC, however the findings of our present study and that of Bagley et al. (1991) confirm that gross haematuria (red discolouration) may cause the UPC to be elevated above 0.5 , and in some instances may be $>2.0$, indicative of glomerular proteinuria. As such, haematuria would need to be considered as a differential in urine samples with UPC $>0.5$ that have gross haematuria.

This study was completed with a limited number of dogs due to ethics and resource restraints. As such further study with larger numbers of both healthy and unwell dogs would be recommended to better extrapolate the results to the general population. The urine colour of these samples was assessed soon after contamination, and analysed shortly thereafter. In practice, storage and transport may affect the urinalysis parameters, so interpretation of colour and haematuria at the point of collection would be recommended (Gunn-Christie et al. 2012). It would be beneficial in stored samples to carefully evaluate whether there are other possible causes of discoloured urine such as myoglobinuria and urinary tract inflammation.

This study has shown that, while blood contamination of $\geq 0.125 \%$ does increase the UPC, if the urine remains yellow (microscopic haematuria) then there is negligible chance that a UPC $>0.5$ will be solely due to the haematuria. In that scenario, attributing the proteinuria present to the haematuria in the sample would be inappropriate. However blood contamination that results in discolouration of the urine sample to red (indicating macroscopic or gross haematuria) could increase the UPC above the abnormal range and would need to be considered as a differential for the proteinuria. Thus, knowledge of urine colour, even if limited to simple colour scores (yellow, discoloured, red) could be utilised to aid interpretation of the UPC in samples with haematuria.

\section{Acknowledgements}

The authors would like to acknowledge the staff of the Massey University veterinary teaching hospital and their dogs for their involvement in this study, New Zealand Veterinary Pathology for providing the equipment on which the samples were assessed. The authors thank Dr Donna Rudd of James Cook University for biochemical advice and Dr Carolyn Gates of Massey University for additional statistical advice. 


\section{References}

Bagley RS, Center SA, Lewis RM, Shin S, Dougherty SA, Randolph JF, Erb H. The effect of experimental cystitis and iatrogenic blood contamination on the urine protein creatinine ratio in the dog. Journal of Veterinary Internal Medicine 5, 66-70, 1991

Bartels H, Bohmer M, Heierli C. Serum creatinine determination without protein precipitation. Clinica Chimica Acta 37, 193-7, 1972

Beatrice L, Nizi F, Callegari D, Paltrinieri S, Zini E, D'Ippolito P, Zatelli A. Comparison of urine protein-to-creatinine ratio in urine samples collected by cystocentesis versus free catch in dogs. Journal of the American Veterinary Medical Association 236, 1221-4, 2010

Brown LD, Cai TT, DasGupta A. Interval estimation for a binomial proportion. Statistical Science $16,101-17,2001$

Brunker J. Protein-losing nephropathy. Compendium on Continuing Education for the Practicing Veterinarian 27, 686-94, 2005

Center SA, Wilkinson E, Smith CA, Erb H, Lewis RM. 24-hour urine protein creatinine ratio in dogs with protein-losing nephropathies. Journal of the American Veterinary Medical Association 187, 820-4, 1985

Cianciolo R, Hokamp J, Nabity M. Advances in the evaluation of canine renal disease. Veterinary Journal 215, 21-9, 2016

Grauer GF. Canine glomerulonephritis: new thoughts on proteinuria and treatment. Journal of Small Animal Practice 46, 469-78, 2005

Grauer GF, Thomas CB, Eicker SW. Estimation of quantitative proteinuria in the dog, using the urine protein-to-creatinine ratio from a random, voided sample. American Journal of Veterinary Research 46, 2116-9, 1985

\section{Gunn-Christie RG, Flatland B, Friedrichs KR, Szladovits B, Harr KE, Ruotsalo K, Knoll JS,} Wamsley HL, Freeman KP. ASVCP quality assurance guidelines: control of preanalytical, analytical, and postanalytical factors for urinalysis, cytology, and clinical chemistry in veterinary laboratories. Veterinary Clinical Pathology 41, 18-26, 2012

Harley L, Langston C. Proteinuria in dogs and cats. Canadian Veterinary Journal-Revue Veterinaire Canadienne 53, 631-8, 2012 
Iwata J, Nishikaze O. New micro-turbidimetric method for determination of protein in cerebrospinal fluid and urine. Clinical Chemistry 25, 1317-9, 1979

Jacob F, Polzin DJ, Osborne CA, Neaton JD, Kirk CA, Allen TA, Swanson LL. Evaluation of the association between initial proteinuria and morbidity rate or death in dogs with naturally occurring chronic renal failure. Journal of the American Veterinary Medical Association 226, $393-400,2005$

Jansen BS, Lumsden JH. Sensitivity of routine tests for urine protein to hemoglobin. Canadian Veterinary Journal 26, 221-3, 1985

Jepson RE. Current understanding of the pathogenesis of progressive chronic kidney disease in cats. Veterinary Clinics of North America-Small Animal Practice 46, 1015-48, 2016

Jergens AE, McCaw DL, Hewett JE. Effects of collection time and food-consumption on the urine protein creatinine ratio in the dog. American Journal of Veterinary Research 48, 1106-9, 1987

Lees GE, Brown SA, Elliott J, Grauer GE, Vaden SL. Assessment and management of proteinuria in dogs and cats: 2004 ACVIM forum consensus statement (small animal). Journal of Veterinary Internal Medicine 19, 377-85, 2005

Luxton RW, Patel P, Keir G, Thompson EJ. A micro-method for measuring total protein in cerebrospinal-fluid by using benzethonium chloride in microtiter plate wells. Clinical Chemistry 35, 1731-4, 1989

Marynissen SJJ, Willems AL, Paepe D, Smets PMY, Picavet P, Duchateau L, Daminet S. Proteinuria in apparently healthy elderly dogs: persistency and comparison between free catch and cystocentesis urine. Journal of Veterinary Internal Medicine 31, 93-101, 2017

McCaw DL, Knapp DW, Hewett JE. Effect of collection time and exercise restriction on the prediction of urine protein excretion, using urine protein creatinine ratio in dogs. American Journal of Veterinary Research 46, 1665-9, 1985

*Stockham S, Scott M. Urinary System. In: Fundamental of Veterinary Clinical Pathology. $2^{\text {nd }}$ Edtn. Pp 415-94. Blackwell Publishing, Ames, IA, USA, 2008

Vaden SL, Pressler BM, Lappin MR, Jensen WA. Effects of urinary tract inflammation and sample blood contamination on urine albumin and total protein concentrations in canine urine samples. Veterinary Clinical Pathology 33, 14-9, 2004 
Vaden SL, Elliott J. Management of proteinuria in dogs and cats with chronic kidney disease.

Veterinary Clinics of North America-Small Animal Practice 46, 1115-30, 2016

\section{Vilhena HCR, Santos RR, Sargo TJ, Lima TB, Dias SS, Pastorinho MR, Queiroga FL,}

Silvestre-Ferreira AC. Urine protein-to-creatinine concentration ratio in samples collected by means of cystocentesis versus manual compression in cats. Journal of the American Veterinary Medical Association 246, 862-7, 2015

White JV, Olivier NB, Reimann K, Johnson C. Use of protein-to-creatinine ratio in a single urine specimen for quantitative estimation of canine proteinuria. Journal of the American Veterinary Medical Association 185, 882-5, 1984

* Non-peer-reviewed

Submitted 30 October 2018

Accepted for publication 24 November 2018

First published online [insert date] 
Table 1. Colour and haematuria category of 18 normal canine urine samples contaminated with $0-5 \%$ blood, and the number of samples with urine protein to creatinine (UPC) ratios $>0.2,>0.5$ and $>2.0$ for each level of contamination.

\begin{tabular}{|c|c|c|c|c|c|}
\hline Blood contamination (\%) & Colour $^{a}$ & Haematuria category ${ }^{b}$ & UPC $\geq 0.2$ & UPC $>0.5$ & UPC > 2.0 \\
\hline 0.00 & Yellow & None & $0(0 \%)$ & $0(0 \%)$ & $0(0 \%)$ \\
\hline 0.125 & Yellow & Microscopic & $1(6 \%)$ & $0(0 \%)$ & $0(0 \%)$ \\
\hline 0.25 & Yellow & Microscopic & $2(11 \%)$ & $0(0 \%)$ & $0(0 \%)$ \\
\hline 0.50 & Yellow/Peach ${ }^{\mathrm{c}}$ & Micro/macroscopic & $7(39 \%)$ & $0(0 \%)$ & $0(0 \%)$ \\
\hline 0.75 & Peach & Macroscopic & $10(56 \%)$ & $0(0 \%)$ & \\
\hline 1.00 & Orange & Macroscopic & $14(78 \%)$ & $2(11 \%)$ & \\
\hline 1.25 & Orange & Macroscopic & 16 (89\%) & $3(17 \%)$ & \\
\hline 1.50 & Orange/Red & Macroscopic & 17 (94\%) & 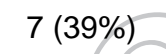 & \\
\hline 1.75 & Orange/Red & Macroscopic & $17(94 \%)$ & $9(50 \%)$ & $0(0 \%)$ \\
\hline 2.00 & Red & Gross & $18(100 \%)$ & & \\
\hline 3.00 & Red & Gross & $18(100 \%)$ & & $0(0 \%)$ \\
\hline 5.00 & Red & Gross & 18 (100\%) & $17(94 \%)$ & $3(17)$ \\
\hline
\end{tabular}

${ }^{a}$ Determined by two observers, resulting in 36 colour scores for each level of contamination

${ }^{\mathrm{b}}$ Based on urine dipstick blood result and sediment examination

${ }^{\mathrm{c}}$ Exactly 18 were scored yellow and 18 were scored peach 
Figure 1. Boxplot showing the distribution of the urine protein to creatinine ratio (UPC) of urine samples from 18 dogs that were contaminated with $0-5 \%$ blood. The median value is indicated by the bold line, the 75th and 25th percentiles are indicated by the upper and lower edges of the boxes, respectively, and the mean by the open circle. The whiskers show the smallest and largest observations that are not suspected outliers, which are the closed circles. Note the $x$-axis is non-linear.

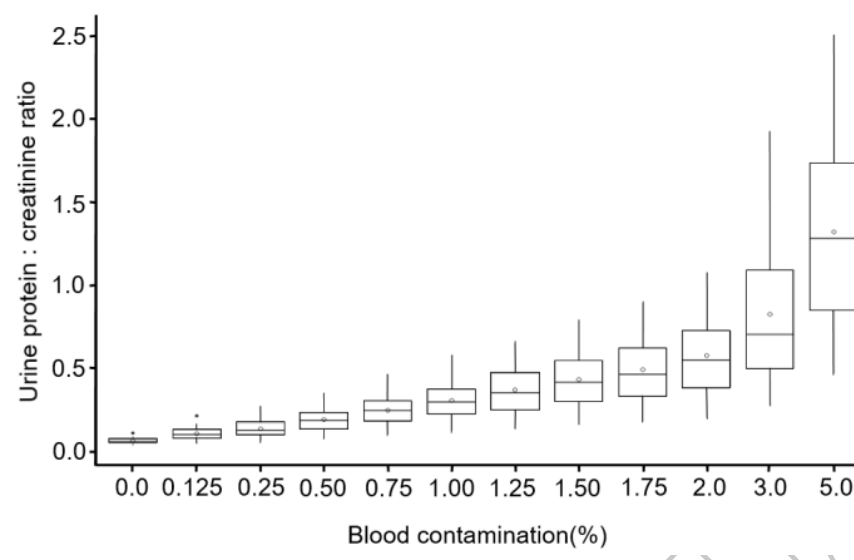

